\title{
Laser Micromachining of Metals with Ultra-Short Pulses: Factors Limiting the Scale-Up Process
}

\author{
Beat Jaeggi $^{* 1}$, Stefan Remund ${ }^{* 1}$, Rene Streubel ${ }^{* 2}$, Bilal Goekce ${ }^{* 2}$, \\ Stefan Barcikowski ${ }^{* 2}$ and Beat Neuenschwander ${ }^{* 1}$ \\ ${ }^{* 1}$ Institute of Applied Laser, Photonics and Surface Technologies ALPS, \\ Bern University of Applied Sciences, Pestalozzistrasse 20, CH-3400 Burgdorf, Switzerland \\ E-mail: beat.neuenschwander@bfh.ch \\ *2 Technical Chemistry I and Center for Nanointegration Duisburg-Essen (CENIDE), \\ University of Duisburg Essen, Universitätsstrasse 7, 45141 Essen, Germany
}

\begin{abstract}
Especially for metals it was shown that the material removal process with ultra-short laser pulses shows an optimum point with highest efficiency i.e. with maximum specific removal rate. A scale-up process should therefore be realized by keeping the applied fluence near this optimum value i.e. by linearly increasing the repetition rate with the average power. In this paper we show that this scale-up is affected by two factors, the heat accumulation and by shielding effects. The latter leads to a significant reduction of the specific removal rate which becomes significant in the case of copper or brass when the repetition rate is increased into the multi-MHz range. In contrast shielding effects are much less pronounced for stainless steel (1.4301) but the surface quality is strongly affected by heat accumulation. However, we show that removal rates in the range of $40 \mathrm{~mm}^{3} / \mathrm{min}$ are achieved for all studied metals with average laser powers above $300 \mathrm{~W}$.
\end{abstract}

DOI: 10.2961/jlmn.2017.03.0016

Keywords: ultra-short laser pulses, micromachining, scale-up and throughput, heat accumulation, plasma-shielding, particle shielding

\section{Introduction}

Ultra-short laser pulses have shown their applicability for high-quality laser micro-machining of metals, semiconductors and insulators in manifold applications. However, to effectively enter into the broad field of industrial applications the demand for high throughput still represents one of the key factors.

It was shown that the specific removal rate (removed volume per energy or removed volume per time and average power) depends on the fluence and hence from the pulse energy itself [1-3]. In the case of a Gaussian beam the specific removal rate as a function of the applied peak fluence $\phi_{0}$ reads in a first approximation:

$$
\frac{d V}{d E}=\frac{d V}{d t \cdot P_{a v}}=\frac{1}{2} \cdot \frac{\delta}{\phi_{0}} \cdot \ln ^{2}\left(\frac{\phi_{0}}{\phi_{t h}}\right)
$$

With $\delta$ the energy penetration depth, $\phi_{t h}$ the threshold fluence and $\phi_{0}=2 E_{p} / \pi w_{0}^{2}$. This function shows a maximum i.e. a highest specific removal rate at an optimum fluence $\phi_{0, o p t}$. The corresponding values are given by:

$$
\left.\frac{d V}{d E}\right|_{\max }=\frac{2}{e^{2}} \cdot \frac{\delta}{\phi_{t h}} ; \phi_{0, o p t}=e^{2} \cdot \phi_{t h}
$$

For a given material the exact values of $\phi_{t h}$ and $\delta$ depend on the pulse duration [4-6] and can additionally depend on the spot size $[7,8]$. Usually the exact ablation process is described with 2 regimes [9], the optical regime at low and the thermal regime at high fluences. In this case a more sophisticated model [10] can be used to describe the specific removal rate (1) as a function of the peak fluence $\phi_{0}$. For copper C12 200 and steel AISI 304 the scale-up process with
10 ps pulses was successfully demonstrated up to about $42 W$ average power by increasing the repetition rate and keeping the peak fluence and the pulse - pulse overlap at a constant value [11]. The corresponding repetition rates amounted 1.21 MHz and 6.83 MHz for copper and steel, respectively. No significant change in the removal rate could be observed up to these high repetition rates. But nowadays ultra-short pulsed laser systems offer average powers up into the $\mathrm{kW}$ range and it has to be investigated how the ablation process can be further scaled.

In [12] it was shown that in case of steel always a constant fraction of the incoming pulse energy is converted to heat. This remaining part leads to heat accumulation [13] becoming stronger at higher pulse - pulse overlap and also higher repetition rate (i.e. higher average power at constant peak fluence) when the overlap is kept constant [14]. It has further been shown, that in case of steel a bumpy low quality surface will appear when the saturated surface temperature just before the next pulse will strike on it exceeds a critical value of about $610^{\circ} \mathrm{C}$.

Shielding effects of ultra-short pulses with a pulse duration of 3.3 ps were investigated in a pump-probe experiment by measuring the transmission perpendicular to the pump pulse at the surface in [15]. Two shielding effects were detected within the ablation process: First plasma shielding starting several $100 \mathrm{ps}$ after the pulse and reaching its strongest shielding after about $5 \mathrm{~ns}$ in case of aluminum steel or copper. Here the transmission drops below $20 \%$. Second, this regime is followed by the so called particle shielding effect where the light is assumed to be Mie-scattered by the particles ejected from the ablation process. This second 
transmission minimum occurs about 200 ns after the pulse. The minimum value of the transmission amounted about $60 \%$ for aluminum and copper and $40 \%$ for steel. The two time intervals where a minimum transmission was observed would correspond to a repetition rate of $200 \mathrm{MHz}(5 \mathrm{~ns})$ and $5 \mathrm{MHz}(200 \mathrm{~ns})$. The presented values for the transmission were obtained at a pump pulse fluence of the $18 \mathrm{~J} / \mathrm{cm}^{2}$ which is far above the optimum peak fluence (2) which amounts about $0.5 \mathrm{~J} / \mathrm{cm}^{2}$ for steel and $3.35 \mathrm{~J} / \mathrm{cm}^{2}$ for copper at a pulse duration of $3 \mathrm{ps}$.

Heat accumulation and particle shielding were also identified as influencing factors for the percussion laser drilling process [16]. But here particle shielding is identified to be present for steel for a repetition rate of $200 \mathrm{kHz}$ and higher for high pulse energies and $500 \mathrm{kHz}$ and higher for low pulse energies. Both repetition rates are far below the expected $5 \mathrm{MHz}$ from [15].

In objection to these observations are the results obtained with double pulses in $[17,18]$. For steel and compared to single pulses the maximum specific removal rate drops by about $20 \%$ for a pulse - pulse interval of $\Delta t_{B}=12 \mathrm{~ns}$ and about $3 \%$ for $\Delta t_{B}=24 n s$. For $36 n s$ and also 60 ns no change to single pulses was observed. The corresponding virtual repetition rates $\left(1 / \Delta t_{B}\right)$ would amount $83 \mathrm{MHz}$ and $41.5 \mathrm{MHz}$ respectively. In contrast to steel a dramatic drop in the specific removal rate of $60 \%$ for a time interval of $12 \mathrm{~ns}(83 \mathrm{MH}), 58 \%$ for $24 \mathrm{~ns}(41.5 \mathrm{MHz}), 53 \%$ for $36 \mathrm{~ns}$ $(27.7 \mathrm{MHz}$ ) and $40 \%$ for $60 \mathrm{~ns}(16.6 \mathrm{MHz})$ was observed at identical speed and overlap. Such strong drops have also been observed for steel with $900 \mathrm{fs}$ pulses, but for time intervals of 2 ns or less [19].

Thus the picture about shielding effects rests partially unclear and its influence onto the specific removal rate at high average powers and high repetition rates has to be determined experimentally for different materials.

\section{Experimental Set-Up}

The used system consisted of

- a high power laser (Amphos 500flex), delivering linearly polarized pulses at $1030 \mathrm{~nm}$ wavelength with an adjustable pulse duration between $900 \mathrm{fs}$ and 5 ps. For the presented experiments the pulse duration was set to 3 ps.

- $\quad$ a high speed polygon scanner with a 12 facet mirror wheel and a duty cycle of $62.5 \%$. The polygon had a telecentric $f=100 \mathrm{~mm}$ objective offering marking speeds up to $480 \mathrm{~m} / \mathrm{s}$. The movement in cross scan direction is realized with an additional galvo-mirror built into the polygon scanner.

The maximum power after the scanner amounted about $300 \mathrm{~W}$ and the repetition rate $f_{\text {rep }}$ could be changed by setting a positive integer (except 2 and 3) as divider for the base repetition rate of $40.5 \mathrm{MHz}$. The chosen repetition rates amounted 2.065 MHz, 5.063 MHz, 8.1 MHz, 10.13 MHz and $40.5 \mathrm{MHz}$. Due to security reasons the laser could only be switched on while the polygon was running. Therefore it was not possible to directly measure the average power and the spot size. The average power was set by defining a percentage of the maximum power which was measured at the installation. Its maximum value directly after the laser system amounted $460 \mathrm{~W}$. To estimate the spot size a comparison with results from experiments performed with a Satsuma
HP2 (set to 3 ps pulse duration) and a repetition rate of $505 \mathrm{kHz}$. For these experiments the spot size was measured $\left(w_{0}=17.2 \mu \mathrm{m}\right)$ and the optimum fluence where the maximum specific removal rate is achieved (2) could exactly be determined. For the experiments with the Amphos system a spot size was assumed and the specific removal rate as a function of the peak fluence, deduced from the average power, the repetition rate and the assumed spot size, is plotted. The spot size was varied until the curve from the Amphos experiments coincided with the one obtained with the Satsuma. This was done for different materials and finally a spot radius of $w_{0}=28 \mu \mathrm{m}$ resulted. Assuming a beam quality factor of $M^{2}=1.3$ this would result in a beam diameter in front of the scanner of about $3 \mathrm{~mm}$ which is a reasonable value as no telescope was installed in front of the polygon line scanner.

Investigated were the removal rates for Copper Cu-DHP (C12 200 in US), stainless steel 1.3401 (AISI 304) and brass (CuZn39Pb2). The samples had a dimension of $50 \times 25 \times 2 \mathrm{~mm}^{3}$ and were placed with a 3 -axes system in the focal plane of the polygon scanner and an average power $P_{a v}$ was set. Then a set of 222 parallel lines with a spacing of $p_{y}=8.4 \mu \mathrm{m}$ was marked, resulting in $1.865 \mathrm{~mm}$ wide areas over the whole sample width as illustrated in Fig. 1. The cross scan movement (jump from line to line) was realized with the internal galvo mirror during the change from one facet to another while the laser was switched off. The distance $p_{x}$ in scan direction was varied from $9 \mu \mathrm{m}$ to $56 \mu \mathrm{m}$ by varying the marking speed $v$ for a given repetition rate $f_{\text {rep: }}$ : $p_{x}=v / f_{\text {rep }}$. Thus the overlap from pulse to pulse was changed from about $85 \%$ down to $0 \%$. The whole sequence of 222 parallel lines was repeated $n_{s l}$ times so that the number of pulses per $\mu \mathrm{m}^{2}$ was constant and amounted 1.700 for steel and 0.638 for copper and brass to obtain similar depths for all materials. The corresponding average number of pulses at a location therefore exceeded 4'100 and 1'500 respectively where the threshold value is no longer influenced by the incubation effect. Then the sample was moved with the linear axes and a new average power was set. A suction tube with a diameter of about $75 \mathrm{~mm}$ was placed just above the sample.

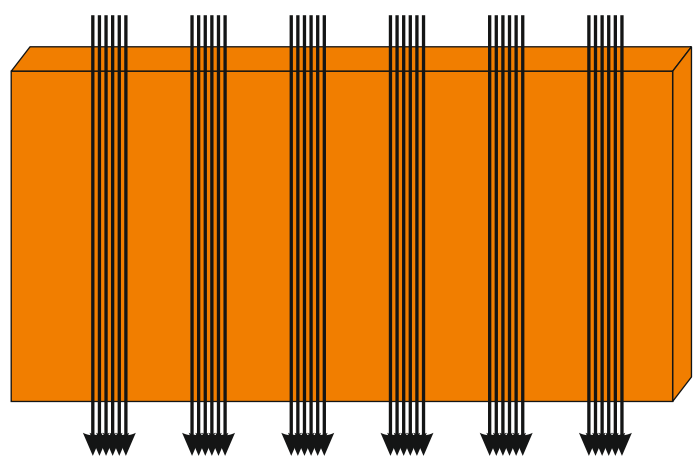

Fig. 1: Sketch of the sample which is machined by different sets of 222 parallel lines. Laser radiation is coming from the top.

The depth $d$ of each machined groove was finally measured with a white light interferometric microscope SmartWLI from gbs. From these data the specific removal rate could be calculated by: 


$$
\frac{d V}{d t \cdot P_{a v}}=\frac{d V}{d E}=\frac{p_{x} \cdot p_{y} \cdot d \cdot f_{\text {rep }}}{n_{s l} \cdot P_{a v}}=\frac{v \cdot p_{y} \cdot d}{n_{s l} \cdot P_{a v}}
$$

It has to be noted here that in contrast to [11] the polygon scanner was not synchronized to the laser and it was not possible to switch on and off the laser during the line marking with one facet. Thus 3D-structuring can't be realized with this set-up. Further the pyramidal error was not corrected finally leading to a wavy surface in the machined grooves as illustrated in Fig. 2. This error could be averaged by starting at every slice with a different facet of the mirror wheel as illustrated in [11] but this averaging was not available for the used high speed polygon.

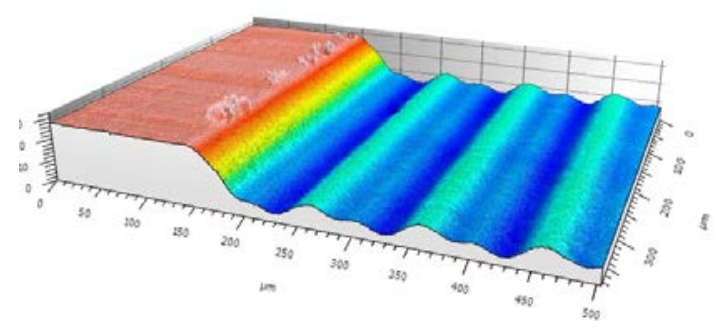

Fig. 2: 3D topography of the machined sample. The waviness is caused by the pyramidal error of the polygon.

\section{Experimental results}

\subsection{Specific Removal Rates for Steel}

The obtained specific removal rate for steel was measured for repetition rates of $8.1 \mathrm{MHz}, 10.13 \mathrm{MHz}$ and $40.5 \mathrm{MHz}$. The results are summarized in Fig. 3 and compared the results obtained by machining squares with the Satsuma system [10]. It is obvious that the maximum rate for the Satsuma experiments, amounting about $3.75 \mu \mathrm{m}^{3} / \mu \mathrm{J}$ is significantly higher than the values of $2.6 \mu \mathrm{m}^{3} / \mu \mathrm{J}$ and $2.2 \mu \mathrm{m}^{3} / \mu \mathrm{J}$ for $8.1 / 10.13 \mathrm{MHz}$ and $40.5 \mathrm{MHz}$, respectively.

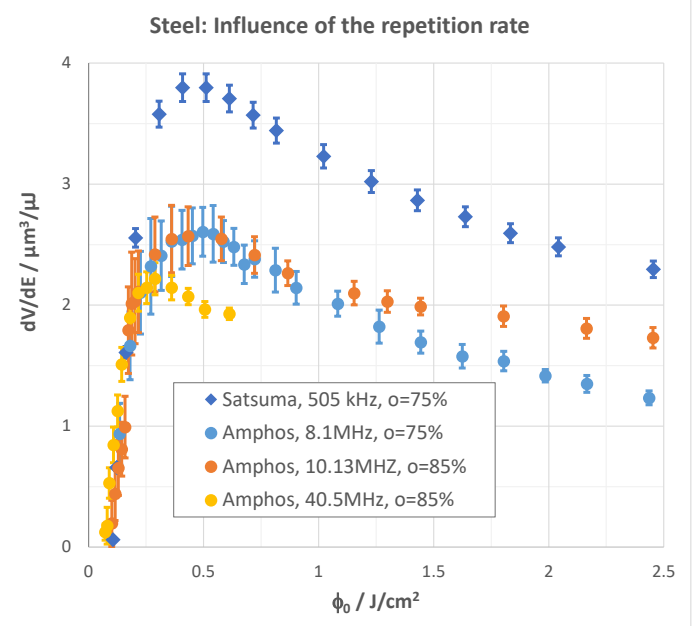

Fig. 3: Specific removal rate of steel as a function of the peak fluence for the high power experiments (dots) and the former Satsuma experiments (dashed line)
The surface quality can explain this behavior. For the repetition rate of $505 \mathrm{kHz}$ the surface was of high quality with a low roughness whereas for $8.1 \mathrm{MHz}$ and $10.13 \mathrm{MHz}$, corresponding to average powers of $50 \mathrm{~W}$ and $55 \mathrm{~W}$ respectively a bumpy surface is observed as shown in the SEM micrograph in Fig. 4a). This effect is even more pronounced for the repetition rate of $40.5 \mathrm{MHz}$ shown in Fig. 4b) and corresponding to an average power of $145 \mathrm{~W}$. This bumpy surface is caused by heat accumulation [13] and could reduce the removal rate as also observed for the cavity formation in steel when too high fluences were applied [17].

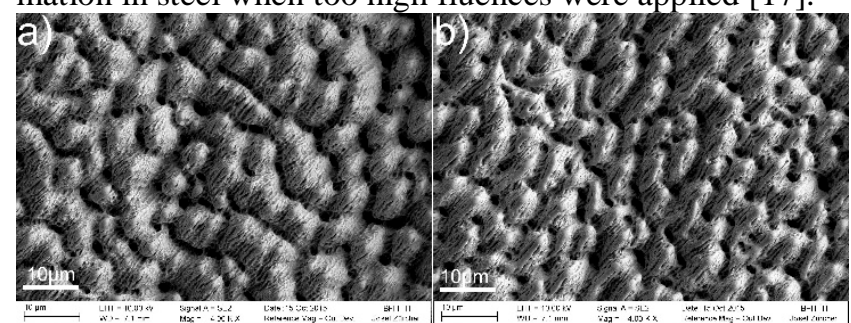

Fig. 4: SEM micrographs of the steel surface a) for a repetition rate of $10.3 \mathrm{MHz}$ and an average power of $55 \mathrm{~W}$ and b) $40.5 \mathrm{MHz}$ and $145 \mathrm{~W}$

To investigate the influence of the surface onto the removal rate the overlap from pulse to pulse was reduced from $85 \%$ to $75 \%, 50 \%, 25 \%$ and $12.5 \%$ at a repetition rate of 10.13 MHz. As illustrated in Fig. 5 no significant difference in the maximum specific removal rate and the fluence where it's located can be detected. SEM micrographs of the corresponding surfaces are shown in Fig. 6. It can clearly be seen that the bumpy surface disappears when the overlap is reduced to $50 \%$ or less. The still observed waviness is due to the pyramidal error of the polygon wheel.

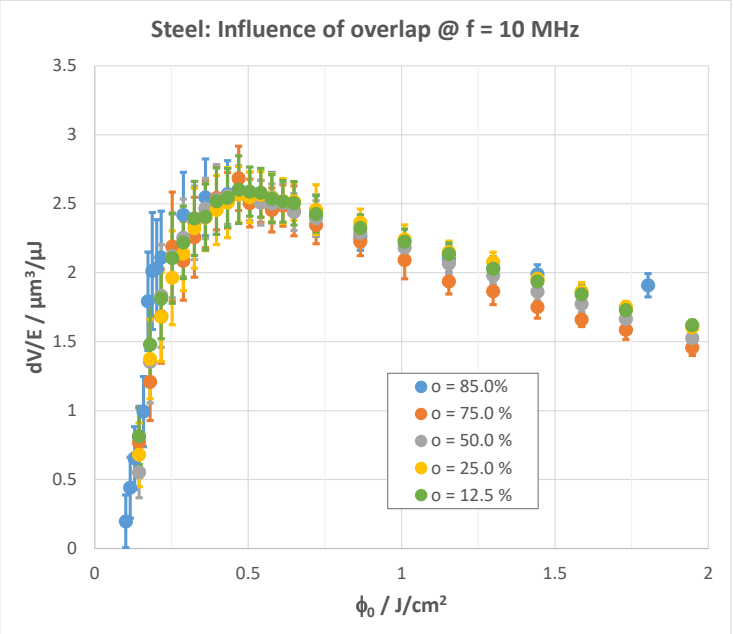

Fig. 5: Specific removal rate for steel and a repetition rate of $10.13 \mathrm{MHz}$. The overlap from pulse to pulse is reduced from $85 \%$ down to $12.5 \%$.

The maximum surface temperature just before the next pulse strikes on the surface was estimated following the analytical model presented in [13]. The results, summarized in Tab. 1, are in line with the predicted critical temperature of about $610^{\circ} \mathrm{C}$ for the appearance of a bumpy surface which should therefore disappear in this situation for an overlap of a little higher than $50 \%$ and less. 


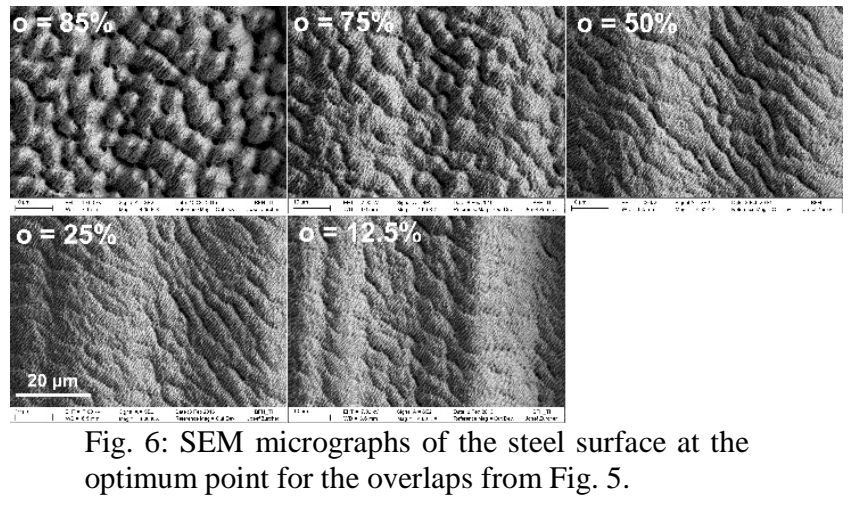

But this reduction of the bumpiness also means that the observed drop in the maximum specific removal rate at higher repetition rates can't be caused by increased surface roughness or heat accumulation and other explanations have to be found.

Table 1: Estimated maximum surface temperature just before the next pulse strikes the surface for steel and the overlaps in Fig. 5.

\begin{tabular}{|l|l|l|l|l|l|}
\hline overlap / \% & 85 & 75 & 50 & 25 & 12.5 \\
\hline$T_{\max } /{ }^{\circ} \mathrm{C}$ & 1166 & 827 & 532 & 477 & 474 \\
\hline
\end{tabular}

In [8] a dependence of the maximum specific removal rate on the spot size was reported for $10 \mathrm{ps}$ pulses. There for $w_{0}=15.5 \mu \mathrm{m}$ a maximum removal rate of $2.17 \mu \mathrm{m}^{3} / \mu \mathrm{J}$ was reported when squares were machined whereas this rate slightly dropped to a value of $2.05 \mu \mathrm{m}^{3} / \mu \mathrm{J}$ for the spot radius of $32.4 \mu \mathrm{m}$. As these spot sizes are in the same range as the spots used in the present experiments the observed difference can't be explained by the different spot size.

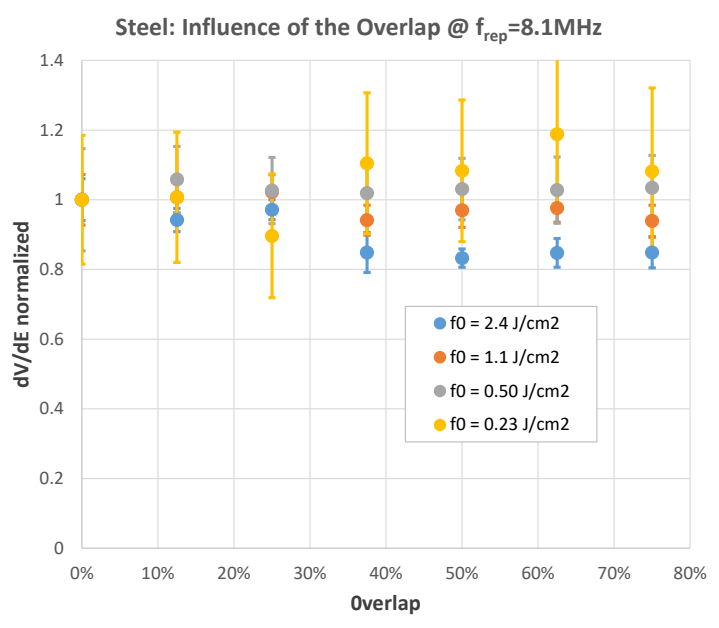

Fig. 7: Specific removal rates as a function of the overlap for steel and a repetition rate of $8.1 \mathrm{MHz}$ and for fixed fluences. The values are normalized to the ones at an overlap of $0 \%$.

Another explanation could be particle- or plasma shielding described in [15]. In this case it is assumed that this effect should be more pronounced for a high overlap and should almost vanish for a full separation of the pulses. Fig. 7 shows the specific removal rate for the peak fluences of $0.23 \mathrm{~J} / \mathrm{cm}^{2}$, $0.5 \mathrm{~J} / \mathrm{cm}^{2}, 1.1 \mathrm{~J} / \mathrm{cm}^{2}$ and $2.4 \mathrm{~J} / \mathrm{cm}^{2}$ normalized to its values obtained for an overlap of $0 \%$. The expected drop in the specific removal rate is observed for the high fluences of $1.1 \mathrm{~J} / \mathrm{cm}^{2}$ and $2.4 \mathrm{~J} / \mathrm{cm}^{2}$ but it amounts only $6 \%$ and $15 \%$, respectively. For the optimum fluence of about $0.5 \mathrm{~J} / \mathrm{cm}^{2}$ and the lower fluence even an increase in the specific removal rate is observed. But one has to have in mind that an increased overlap also leads to heat accumulation and higher surface temperatures as illustrated in tab. 1 . This effect could therefore directly compensate the expected increased shielding for higher overlaps.

The highest absolute removal rate of $35.3 \mathrm{~mm} / \mathrm{min}$ was obtained for $40.5 \mathrm{MHz}$ repetition rate at the highest peak fluence corresponding to an average power of $306 \mathrm{~W}$. The corresponding bumpy surface is shown in Fig. 5b).

\subsection{Specific Removal Rates for Copper}

The specific removal rate for copper was measured for repetition rates of $2 \mathrm{MHz}, 5 \mathrm{MHz}, 8.1 \mathrm{MHz}, 10.13 \mathrm{MHz}$ and $40.5 \mathrm{MHz}$. For comparison again the results from the Satsuma experiments at $0.5 \mathrm{MHz}$ were also added to Fig. 8 . Again we can observe a drop in the maximum specific removal rate which is more pronounced in the $\mathrm{MHz}$ regime than for steel and is dramatic for the highest repetition rate of $40.5 \mathrm{MHz}$. The corresponding maximum specific removal rates amounted $2.86 \mu \mathrm{m}^{3} / \mu \mathrm{J}, 1.97 \mu \mathrm{m}^{3} / \mu \mathrm{J}, 1.81 \mu \mathrm{m}^{3} / \mu \mathrm{J}$, $1.60 \mu \mathrm{m}^{3} / \mu \mathrm{J}, 1.45 \mu \mathrm{m}^{3} / \mu \mathrm{J}$ and $0.75 \mu \mathrm{m}^{3} / \mu \mathrm{J}$. As for steel also copper shows a significant drop in the maximum specific removal rate from the Satsuma experiments with $w_{0}=17.2 \mu \mathrm{m}$ compared to the high power experiments with $w_{0}=28 \mu \mathrm{m}$. Also here the observed change in the maximum specific removal rate for two similar spot sizes and $10 \mathrm{ps}$ pulses was only a few \%, as reported in [8], and can't explain this change.

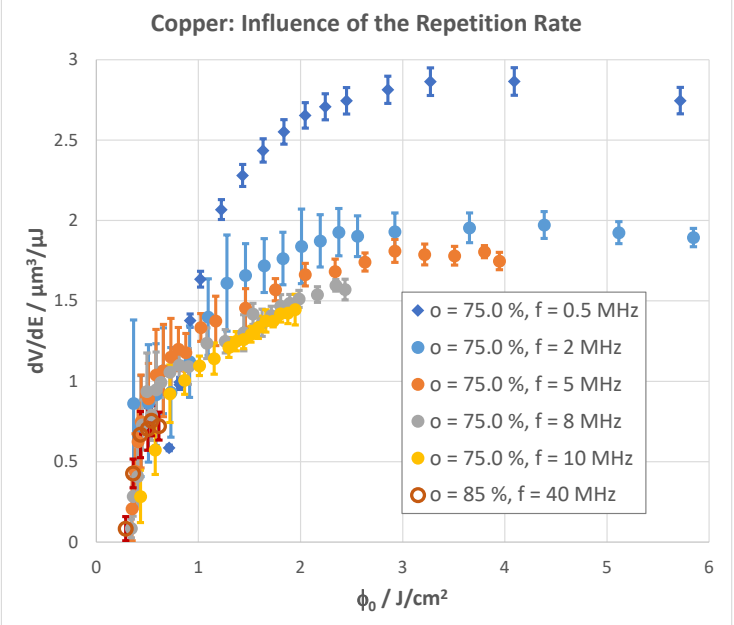

Fig. 8: Specific removal rate of copper as a function of the peak fluence for the high power experiments (dots) and the former Satsuma experiments (diamonds)

In contrast to steel also the influence of the overlap is much more pronounced as illustrated in Fig. 9. Here the overlap was reduced from $85 \%$ down to $0 \%$ for a repetition rate of $5 \mathrm{MHz}$.

For the fixed fluences of $1 \mathrm{~J} / \mathrm{cm}^{2}, 2.6 \mathrm{~J} / \mathrm{cm}^{2}$ (near optimum point), $3.9 \mathrm{~J} / \mathrm{cm}^{2}$ and the maximum values as well the specific removal rate is shown as a function of the overlap in Fig 10. 
For the two higher fluences and the maximum values the specific removal rate rests almost constant when the overlap is increased from $0 \%$ to $37.5 \%$. Then it drops and reaches its lowest value at an overlap of $62.5 \%$ and increases again and reaches a value at an overlap of $75 \%$ which exceeds the one obtained at $0 \%$ overlap. Again shielding effects are expected to cause the observed drop and heat accumulation will finally lead to higher values observed at higher overlaps.

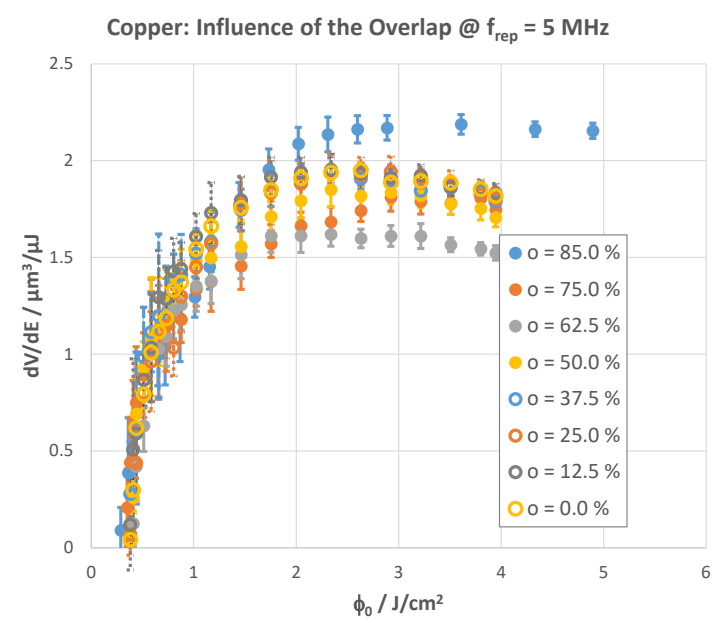

Fig. 9: Specific removal rate as a function of the peak fluence for copper, a repetition rate of $5 \mathrm{MHz}$ and different overlaps.

For the lower fluence of about $1 \mathrm{~J} / \mathrm{cm}^{2}$ a constant drop of the specific removal rate with increasing overlap is observed. At this lower power it is assumed that heat accumulation can no longer compensate the reduction caused by increased shielding effects.

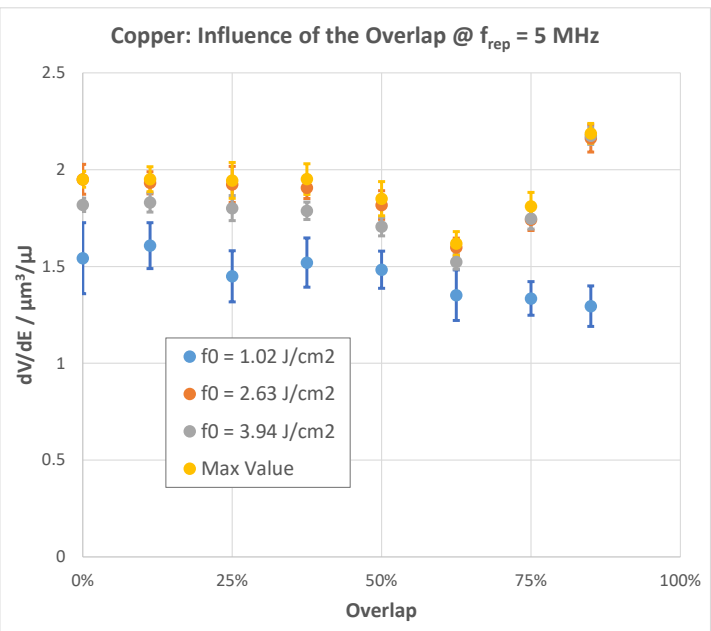

Fig. 10: Specific removal rate for copper as a function of the overlap. Beside the maximum values also the specific removal rate for three specific fluences are shown.

The highest absolute value of the removal rate for copper amounted $39.5 \mathrm{~mm}^{3} / \mathrm{min}$ and was obtained at a repetition rate of $5 \mathrm{MHz}$, an overlap of $85 \%$ and at the maximum average power of $306 \mathrm{~W}$. At this point the surface quality is still high as illustrated in Fig. 11.

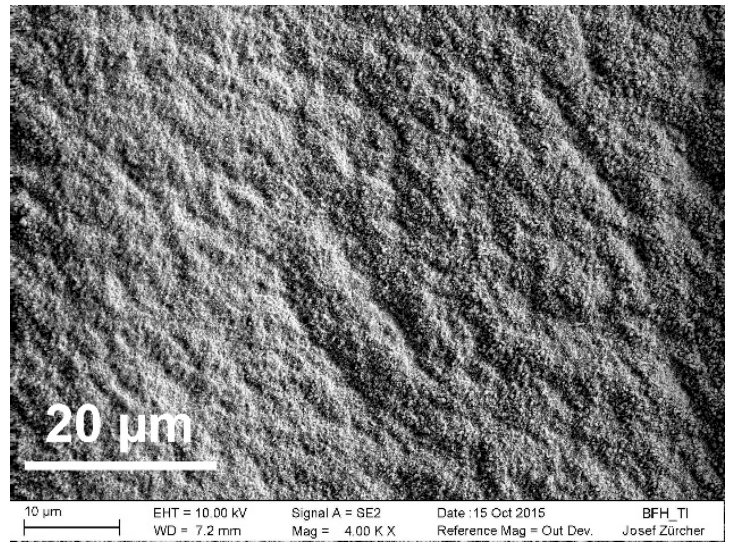

Fig. 11: SEM micrograph of the copper surface machined with a repetition rate of $5 \mathrm{MHz}$ and an average power of $306 \mathrm{~W}$.

\subsection{Specific Removal Rates for Brass}

As for copper, the specific removal rate for brass was measured for repetition rates of $2 \mathrm{MHz}, 5 \mathrm{MHz}, 8.1 \mathrm{MHz}$ and $10.13 \mathrm{MHz}$ and compared with the results from the former Satsuma experiments at $0.5 \mathrm{MHz}$. The specific removal rates as a function of the peak fluence and at an overlap of $75 \%$ are summarized in Fig. 12. Again a strong drop is observed between the repetition rate of $0.5 \mathrm{MHz}$ and the high power experiments in the $\mathrm{MHz}$ range. The corresponding maximum removal rates amounted $4.37 \mu \mathrm{m}^{3} / \mu \mathrm{J}, \quad 3.15 \mu \mathrm{m}^{3} / \mu \mathrm{J}$, $3.09 \mu \mathrm{m}^{3} / \mu \mathrm{J}, \quad 2.83 \mu \mathrm{m}^{3} / \mu \mathrm{J}$ and $2.71 \mu \mathrm{m}^{3} / \mu \mathrm{J}$ and were achieved at peak fluences of $1.36 \mathrm{~J} / \mathrm{cm}^{2}, 2.56 \mathrm{~J} / \mathrm{cm}^{2}$, $1.75 \mathrm{~J} / \mathrm{cm}^{2}, 1.80 \mathrm{~J} / \mathrm{cm}^{2}$ and $1.44 \mathrm{~J} / \mathrm{cm}^{2}$. If only the values for the high power experiments are considered it seems as the fluence, where the maximum specific removal rate is achieved, shifts to lower values when the repetition rate and therefore the average power is increased.

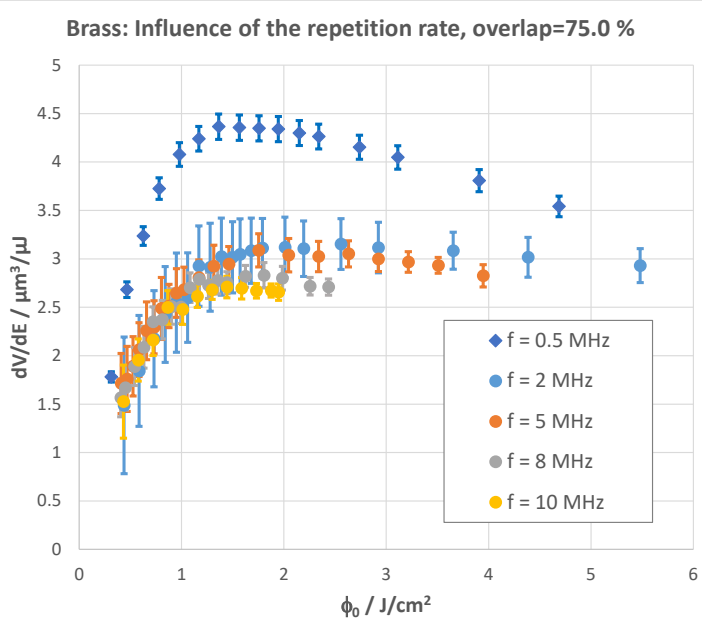

Fig. 12: Specific removal rate of brass as a function of the peak fluence for the high power experiments (dots) and the former Satsuma experiments (diamonds)

The specific removal rate as a function of the overlap, varied from $25 \%$ to $75 \%$, for the fixed fluences $0.58 \mathrm{~J} / \mathrm{cm}^{2}$, $1.15 \mathrm{~J} / \mathrm{cm}^{2}, 1.44 \mathrm{~J} / \mathrm{cm}^{2}$ and $1.95 \mathrm{~J} / \mathrm{cm}^{2}$ are shown for the repetition rate of $10.1 \mathrm{MHz}$ in Fig. 13. For the three high fluence values the specific removal rate rest almost constant whereas for the lowest fluence a small trend to lower values can be observed. 


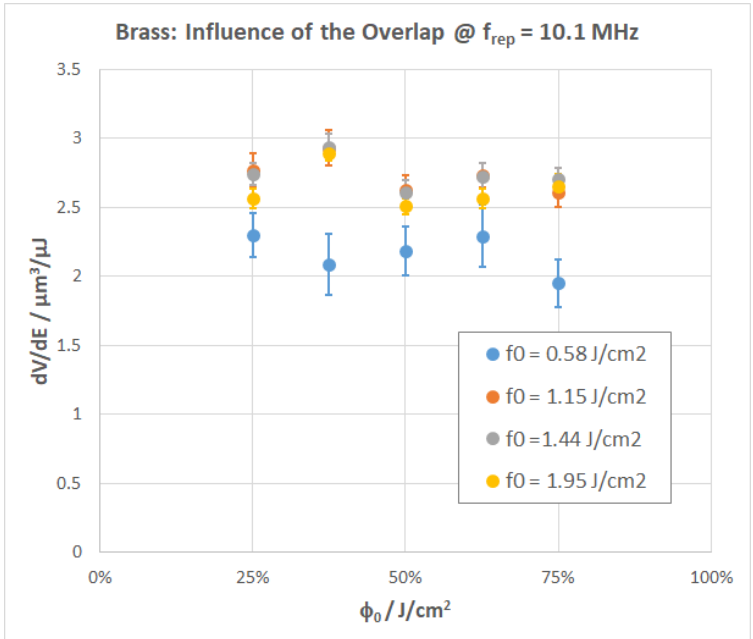

Fig. 13: Specific removal rate for brass as a function of the overlap for four specific fluences.

An absolute removal rate of $41 \mathrm{~mm}^{3} / \mathrm{min}$ and $39.5 \mathrm{~mm}^{3} / \mathrm{min}$ was achieved for the repetition rates of $5 \mathrm{MHz}$ and $8.1 \mathrm{MHz}$, respectively. For the brass experiments the maximum average power amounted $243 \mathrm{~W}$ and not $306 \mathrm{~W}$ as for copper and steel. In both cases a good surface quality was achieved as illustrated in Fig. 14. The small holes detected on the surface are expected to be caused by lead inclusions in the used brass.

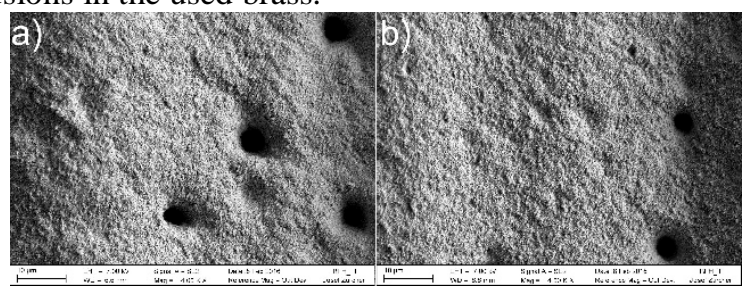

Fig. 14: SEM micrographs from the brass surfaces machined with $5 \mathrm{MHz}$ a) and $8.1 \mathrm{MHz}$ b) with an average power of $243 \mathrm{~W}$. The detected holes are expected to be caused by the lead inclusions of brass.

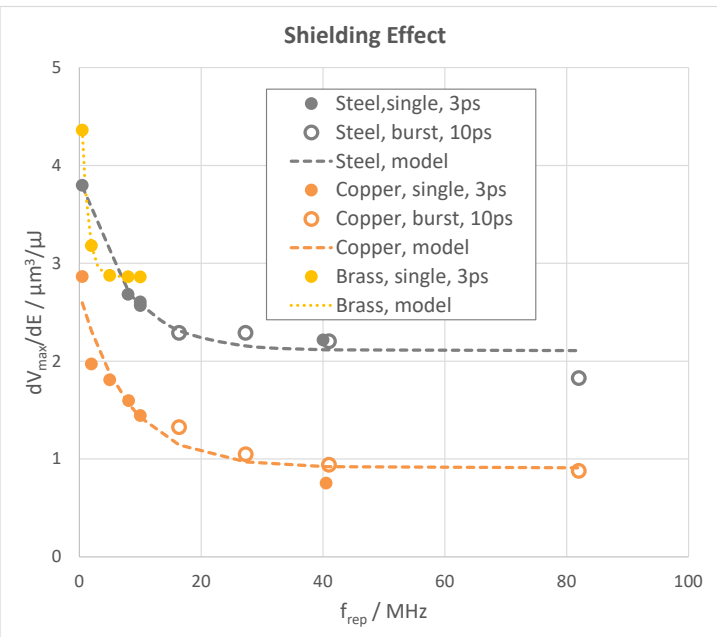

Fig. 15: Specific removal rates obtained from the presented high power experiments (filled circles) combined with the results obtained for double pulse experiments with $10 \mathrm{ps}$ pulses (rings) in [17]. The dashed lines represents the least square fit according to (4).

\section{Shielding}

For all investigated materials a drop of the maximum specific removal rate could be observed when the repetition rate is increased into the $\mathrm{MHz}$-regime. This decrease is more pronounced for copper and brass and is reduced for steel where also a repetition rate of $40.5 \mathrm{MHz}$ could be applied without high losses. In principle the results of this work could be combined with the results from burst experiments presented in [17] where the spot radius was $16 \mu \mathrm{m}$, the repetition rate amounted $200 \mathrm{kHz}$ and the overlap was set to $75 \%$. In these experiments a significant reduction of the specific removal rate for a double pulse was observed. This reduction is also expected to be caused by shielding effects. A double pulse with a time interval of $\Delta t_{B}$ could in a first approximation be considered as a laser with the repetition rate of $f_{\text {rep }}=1 / \Delta t_{B}$. Fig. 15 shows the combination of these results for copper and steel with the ones presented here. For brass no multi-pulse burst experiments were performed.
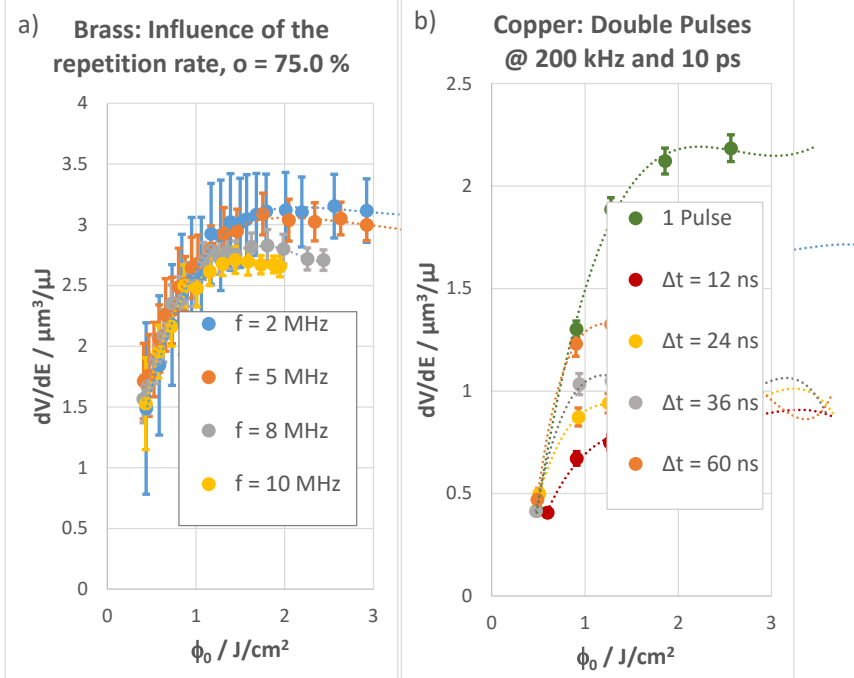

Fig. 16: Specific removal rate as a function of the peak fluence at low fluence values. Left: For brass and different repetition rates. Right: For copper and double pulses with different time interval. The dashed lines represent guides to the eye.

If it is assumed that the removed material forms a plume of particles and/or plasma its density will decrease in time because it is expanding from the surface. In a first approximation, at least for $w_{0}=28 \mu \mathrm{m}$, it is assumed that this plume behaves like a cylinder only expanding in height. It is further assumed that the expansion speed of the plume drops exponentially in time and that the transmission through the plume is inverse proportional to the averaged density in this cylinder. An analysis of the specific removal rate as function of the peak fluence for low fluence values shows that the corresponding curves first follows almost the same trend for all different repetition rates or time interval between the pulses in case of a double pulse. This is illustrated in Fig. 16a) for brass machined in the $\mathrm{MHz}$ regime with the 3ps pulses and in Fig. 16b) for copper machined with double pulses with a pulse length of $10 \mathrm{ps}$. The dashed lines, representing guides to the eye, shows that the trend of the specific removal rate begins to deviate when a certain peak fluence is reached. This "deviation fluence" drops with increasing repetition rate or decreasing time interval. As a higher peak fluence is going with a higher ablated volume per pulse and a higher 
repetition rate or smaller time interval with a shorter expansion time this indicates that a minimum density in the plume has to be reached before shielding can take place. Therefore it is further assumed that the transmission can't become smaller than a minimum value. Then it can be shown that the specific removal rate can be expressed as a function of the repetition rate by:

$$
\frac{d V}{d E}\left(f_{\text {rep }}\right)=\left.\frac{d V}{d E}\right|_{\min }+\Delta \rho \cdot e^{-k \cdot f_{\text {rep }}}
$$

The corresponding least square fits are shown as dashed lines in Fig. 15.

\section{Conclusion}

Experiments with a high power ultra-short pulsed laser system have been performed to investigate the scale-up of the ablation process for steel, copper and brass. All materials were machined in the $\mathrm{MHz}$ range up to an average power of $243 \mathrm{~W}$ for brass and $306 \mathrm{~W}$ for steel and copper. For steel heat accumulation leads to a bumpy surface and limits the scale-up process if a high surface quality is demanded.

For copper and brass removal rates up to $40 \mathrm{~mm}^{3} / \mathrm{min}$ with good surface quality were demonstrated. But it has to be mentioned here that the used repetition rates were $5 \mathrm{MHz}$ and higher and the polygon was not synchronized to the laser pulse train. Thus a real 2D or 3D structuring was not possible and the possibility of single pulse switching, which would be demanded for such applications, will be a real challenge at such high repetition rates and average powers. If this could be realized, it is expected that, especially for brass where the maximum average power was only $243 \mathrm{~W}$, removal rates in the range of $1 \mathrm{~mm}^{3} / \mathrm{s}$ could be achievable.

However, for all investigated materials the results show that shielding effects are limiting the scale-up process when high repetition rates have to be used. Even if heat accumulation could partially compensate the shielding effect, as indicated by the experiments with carrying overlap, shielding will become the dominant effect for high repetition rates.

A simple model was presented explaining the trend of the maximum specific removal rate as a function of the repetition rate or the inverse time difference between two pulses. Even this model fits well the experimental data the assumptions on which it is based are a strong simplification of the real processes going on. Further it has also to be mentioned here that a double pulse do not really correspond to a single pulses at high repetition. However, in both situations shielding is expected to cause the observed drop in the maximum specific removal rate and it becomes stronger the higher the repetition rate or the smaller the time interval between two pulses become.In principal this problem can be solved by increasing separation between the pulses, but this would demand even higher marking speeds up to several $1000 \mathrm{~m} / \mathrm{s}$. Also a stronger suction system, eventually with a fast gas jet, could help to reduce the shielding effect. Experiments where the target was placed in a fast flowing liquid [20] have shown an increase in the maximum specific removal rate for copper when the repetition rate is raised from $1.2 \mathrm{MHz}$ up to 10.1 MHz But in these experiments the fluence where the maximum specific removal rate is obtained significantly decreases from $1.2 \mathrm{~J} / \mathrm{cm}^{2}$ at $1.2 \mathrm{MHz}$ down to $0.3 \mathrm{~J} / \mathrm{cm}^{2}$ at 10.1 MHz. Thus much less volume and mass per pulse is removed at the high repetition rate and the observed increase in the maximum specific removal rate could also be caused by this fact. Therefor additional experiments will be needed to gain a clearer picture of the influence of shielding effects.

\section{Acknowledgments and Appendixes}

This work was supported by the European Union via the project APPOLO (GA 609355), the Deutsche Forschungsgemeinschaft (DFG) (INST 20876/134-1 FUGG) and the Bundesministerium für Bildung und Forschung (BMBF) (03SF0497C)

The authors thank Josef Zuercher for the help with the SEM micrographs and Urs Hunziker for his work in the mechanical workshop.

\section{References}

[1] G. Raciukaitis, M. Brikas, P. Gecys, B. Voisiat, M. Gedvilas: J. Laser Micro/Nanoengin., 4, (2009) 186.

[2] B. Neuenschwander, G. Bucher, Ch. Nussbaum, B. Joss, M. Muralt, U. Hunziker, P. Schuetz: Proc. of SPIE, 7584, (2010) 75840R.

[3] B. Neuenschwander, B. Jaeggi, M. Schmid: Phys. Proceedia, 41, (2013) 794.

[4] M. Schmid, B. Neuenschwander, V. Romano, B. Jaeggi, U.W. Hunziker: Proc. of SPIE, 7920, (2011) 792009.

[5] J. Lopez, R. Kling, R, Torres, A. Lidolff, M. Delaigue, S. Ricaud, C Hoenninger, E. Mottay: Proc. of SPIE, 8243, (2012) 824300.

[6] B. Jaeggi, B. Neuenschwander, U. Hunziker, J. Zuercher, T. Meier, M. Zimmermann, K.H. Selbmann, G. Hennig: Proc. of SPIE, 8243, (2012) 82430K.

[7] O. Armbruster, A. Naghilou, M. Kitzler, W. Kautek: Appl. Surf. Sci., 396, (2017) 1736.

[8] B. Lauer, B. Jaeggi, Y. Zhang, B. Neuenschwander: Proc. of ICALEO 2015, Paper M701.

[9] B.N. Chichov, C. Momma, S. Nolte, F. van Alvensleben and A. Tünnermann: Appl. Phys. A, 63, (1996) 109.

[10]B. Jaeggi, B. Neuenschwander, S, Remund, Th. Kramer: Proc. of SPIE, 10091, (2017) 100910J.

[11]B. Jaeggi, B. Neuenschwander, M. Zimmermann, L. Penning, R. De Loor, K. Weingarten, A. Oehler: Proc. of SPIE, 8967, (2014) 89670Q.

[12]F. Bauer, A. Michalowsi, St. Nolte: J. Laser Micro/Nanoengin., 10, (2015) 325.

[13]F. Bauer, A. Michalowski, Th. Kiedrowski, St. Nolte: Opt. Express, 23, (2015) 1035.

[14]B. Neuenschwander, B. Jaeggi, M. Zimmermann, V. Markovic, B. Resan, K. Weingarten, R. deLoor, L. Penning: J. Laser Appl., 28, (2016).

[15] J. Koenig, S. Nolte, A. Tuennermann: Opt. Express, 13, (2005) 10597

[16]A. Ancona, S. Doering, C. Jauregui, F. Roeser, J. Limpert, S. Nolte, A. Tuennermann: Opt. Lett., 34, (2009) 3304.

[17]B. Neuenschwander, Th. Kramer, B. Lauer, B. Jaeggi: Proc. of SPIE, 9350, (2015) 93500U.

[18]Th. Kramer, Y. Zhang, St. Remund, B. Jaeggi, A. Michalowski, L. Grad, B. Neuenschwander: J. Laser Micro/Nanoengin., 12, (2017) 107.

[19] M. Sailer, F. Bauer, J. Kleiner, M. Kaiser, Proc. of Lasers in Manufacturing Conference, (2015).

[20]R. Streubel, S. Barcikowski, B. Gökce: Opt. Lett., 41, (2016) 1486 